\title{
Superposition rule for light scattering by a composite particle
}

Min Xu

Fairfield University, mxu@fairfield.edu

Follow this and additional works at: https://digitalcommons.fairfield.edu/physics-facultypubs (C) 2006 The Optical Society. This paper was published in Optics letters and is made available as an electronic reprint with the permission of OSA. The paper can be found at the following URL on the OSA website:

http://www.opticsinfobase.org/ol/abstract.cfm?URI=ol-31-22-3223

Systematic or multiple reproduction or distribution to multiple locations via electronic or other means is prohibited and is subject to penalties under law.

\section{Peer Reviewed}

\section{Repository Citation}

Xu, Min, "Superposition rule for light scattering by a composite particle" (2006). Physics Faculty Publications. 13.

https://digitalcommons.fairfield.edu/physics-facultypubs/13

\section{Published Citation}

Min Xu. Superposition rule for light scattering by a composite particle. Optics Letters, 31:3223-3225, 2006. http://www.opticsinfobase.org/ol/abstract.cfm?URI=ol-31-22-3223

This item has been accepted for inclusion in DigitalCommons@Fairfield by an authorized administrator of DigitalCommons@Fairfield. It is brought to you by DigitalCommons@Fairfield with permission from the rightsholder(s) and is protected by copyright and/or related rights. You are free to use this item in any way that is permitted by the copyright and related rights legislation that applies to your use. For other uses, you need to obtain permission from the rights-holder(s) directly, unless additional rights are indicated by a Creative Commons license in the record and/or on the work itself. For more information, please contact digitalcommons@fairfield.edu. 


\title{
Superposition rule for light scattering by a composite particle
}

\author{
Min Xu \\ Department of Physics, Fairfield University, Fairfold, Connecticut ob430 \\ Received July 17, 2006; revised September 1, 2006; accepted September 2, 2006; \\ posted September 6, 2006 (Doc. ID 73097); published October 26, 2006
}

\begin{abstract}
A superposition rule for light scattering by composite particles is presented that expresses the scattering amplitude of a composite particle as a superposition of that of the host particle and those of the shadowed inclusions. The superposition rule is derived for a soft composite particle but also provides insight into light scattering by a general composite scatterer. Favorable comparison with an exact numerical method demonstrates the usefulness of the rule in analyzing light scattering by composite particles such as biological cells. (6) 2006 Optical Society of America

OCIS codes: $290.4020,290.5850,170.4580,170.1580,010.1310$.
\end{abstract}

Light interaction with small particles is the foundation for remote sensing and biomedical optical spectroscopy and imaging. Mie theory ${ }^{1}$ is widely used for spherical uniform particles. For nonspherical particles or composite particles, there is no analytical exact solution available, and numerical methods are commonly required. ${ }^{2}$ Light scattering from composite particles such as aerosol particles ${ }^{3}$ and cells, ${ }^{4}$ which contain internal structures, is of particular interest in atmospheric science and biomedical applications. For example, the change of the nuclear structure in a cell during neoplastic progression may be probed optically. ${ }^{5}$ Computation of light seattering by a composite particle with inclusions is much more difficult than that for a uniform particle. ${ }^{2,4,6,7}$ The effect of the presence of inclusions on the light scattering property is hard to quantify. In this Letter, a superposition rule for light scattering by composite particles is presented. The superposition rule shows that light scattering by a composite particle can be constructed from a superposition of light scattering by the host particle and those by the shadowed inclusions. This rule applies to composite particles that are optically soft (relative refractive index $|m-1| \ll 1$ ) when the shadowing of the host particle on inclusions is not far from uniformity. However, it also yields valuable insight into light scattering by a general composite particle.

Light scattering by a soft particle can be well described by the anomalous diffraction theory (ADT) of van de Hulst. $1,8,9$ The applicability of ADT extends to nonsoft particles. ${ }^{10,11}$ The scattering amplitude function in ADT is given by

$$
S(\boldsymbol{q})=\frac{k^{2}}{2 \pi} \int\left\{1-\exp [-i p(\xi, \eta)] e^{-i\left(\xi q+\eta q_{\xi} \eta\right)} \mathrm{d} \xi \mathrm{d} \eta\right.
$$

where the integration of $\xi$ and $\eta$ is over the area of the projection of the particle in the direction ( $z$ axis) of the incident light, $p(\xi, \eta)=k \int d z[m(\xi, \eta, z)-1]$ is the phase delay of the ray piercing the particle at the position $(\xi, \eta), \quad q=\left(q_{\xi}, q_{n}, 0\right)=q(\cos \phi, \sin \phi, 0)$ is the wave-vector transfer with a magnitude $2 k \sin (\theta / 2), k$ is the wavenumber, and $\theta$, $\phi$ are the polar and azimuthal angles of scattering, respectively. For a composite particle of the refractive index $m_{0}$ containing a region (nucleus) of refractive index $m_{1}=m_{0}+\delta m$, the phase delay $p$ can be written as the sum of two parts: the phase delay in the host particle $p_{0}$ $=k \int \mathrm{d} z\left[m_{0}(\xi, \eta, z)-1\right]$ and the additional phase delay due to the nucleus $\delta p(\xi, \eta)=k \int \mathrm{d} z \delta m(\xi, \eta, z)$ [see Fig. 1(a)]. As a consequence, the scattering amplitude function can be written as

$$
S(\boldsymbol{q})=S_{0}(\boldsymbol{q})+\exp \left(-i \boldsymbol{r}_{c} \cdot \boldsymbol{q}\right) f_{c} S_{n}(\boldsymbol{q})
$$

where $S_{0}$ and $S_{n}$ are the scattering amplitude functions for the host particle of refractive index $m_{0}$ and the shadowed nucleus of refractive index $\delta m+1$, respectively, $\mathbf{r}_{c}=\left(\xi_{c}, \eta_{c}, z_{c}\right)$ is the position of the center of the nucleus relative to the center of the host particle, $f_{c}$ is an operator, and $f_{c} S_{n}(\theta)$ is defined by

$$
\begin{aligned}
f_{c} S_{n}(\boldsymbol{q})= & \frac{k^{2}}{2 \pi} \int f_{c}\left(\xi^{\prime}, \eta^{\prime}\right)\left\{1-\exp \left[-i \delta p\left(\xi^{\prime}, \eta^{\prime}\right)\right]\right\} \\
& \times e^{-i\left(\xi^{\prime} q_{\xi} \eta^{\prime} q^{\prime}\right.} \mathrm{d} \xi^{\prime} \mathrm{d} \eta^{\prime},
\end{aligned}
$$

with $f_{c}\left(\xi^{\prime}, \eta^{\prime}\right)=\exp \left[-i p_{0}\left(\xi^{\prime}+\xi_{c}, \eta^{\prime}+\eta_{c}\right)\right]$.

The essence of ADT is that light penetrates straight through the scatterer with only a minimal bending of the ray. Hence light scattering by a composite particle is equivalent to that by two individual entities (the host particle and the nucleus), where the nucleus outside is shadowed by the host particle [see Fig. 1(b)]. If the maximum difference $(\Delta \rho)_{\text {max }}$ in the phase delay $p_{0}\left(\xi^{\prime}+\xi_{c}, \eta^{\prime}+\eta_{c}\right)$ of the illuminating light on the nucleus due to the host particle is small, then we can replace $f_{c}$ in Eq. (3) by a constant $\exp (-i \bar{\rho})$, where $\bar{p}$ is the average phase delay caused by the host particle. If $(\Delta p)_{\max } \geqslant 1$, different regions of the shadowed nucleus are illuminated by light in phase and out of phase alternatively. Since light scattering from volumes of the nucleus illuminated by light out of phase tends to cancel each other, light scattering by the shadowed nucleus is then much suppressed and quite different from that found when it is standalone. 


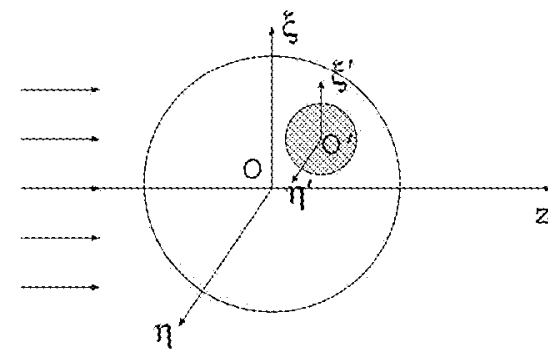

(a)

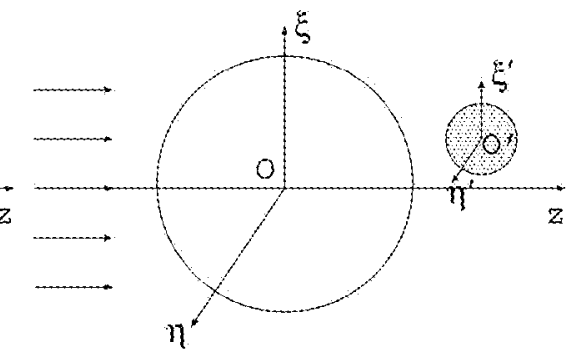

(b)

Fig. 1. (a) Composite scatterer illuminated by an incident beam along the $z$ axis. (b) Equivalent configuration with an outside nucleus shadowed by the host particle. The shadowed nucleus has a modified refractive index.

Based on the above observation, the superposition rule asserts that the light scattering amplitude for a composite particle can be expressed as a superposition of those of the host particle and the shadowed nucleus:

$$
S(\boldsymbol{q})=S_{n}(\boldsymbol{q})+\exp \left(-i \mathbf{r}_{i} \cdot \boldsymbol{q}-i \bar{\rho}\right) S_{n}(\boldsymbol{q}),
$$

where $\bar{\rho}$ is the average phase delay seen by the nucleus due to the host particle as long as $(\Delta p)_{\max }$ $<1$. The shadowed nucleus is the same as the real nucleus except that its relative refractive index is replaced by $m_{1}-m_{0}+1$. If the host particle contains multiple inclusions, Eq. (4) becomes

$$
S(\boldsymbol{q})=S_{0}(\boldsymbol{q})+\sum_{j} \exp \left(-i \mathbf{x}_{i_{j}} \cdot \boldsymbol{q}-i \bar{p}_{j}\right) S_{j}(\boldsymbol{q})
$$

where $\bar{p}_{j}$ is the average phase delay seen by the $j$ th inclusion centered at $\boldsymbol{r}_{c_{j}}$ due to the host particle (and possibly other inclusions), and $S_{j}$ is the scattering amplitude function for the $j$ th shadowed inclusion (with a modified refractive index) provided $(\Delta \rho)_{\max }$ $<1$ is satisfied by each inclusion. The superposition rule extends to the scattering amplitude matrix ${ }^{1}$ in which $S$ in Eqs. (4) and (5) is a $2 \times 2$ matrix. After obtaining the scattering amplitude of a composite particle, its scattering properties such as optical efficiencies, phase function, and Muller matrix will be fully determined.

The application of this rule for a concentric sphere will be demonstrated. Denote the radius of the host particle $a$ and the radius of the nucleus $b$. The average phase delay is $\bar{\rho}=\frac{2}{3} \rho_{\max } \gamma^{-2}\left[1-\left(1-\gamma^{2}\right)^{3 / 2}\right]$, and the maximum phase delay difference is $(\Delta \rho)_{\max }=\frac{1}{2} \rho_{\max } \gamma^{2}$, where $\rho_{\max }=2 k\left(m_{0}-1\right) a$ and $\gamma=b / a$. The superposition rule can be applied when $\frac{1}{2} \rho_{\max } \gamma^{2}<1$.

Figure 2 displays the light extinction efficiencies for various concentric spheres computed exactly ${ }^{12}$ and approximately by using the superposition rule, where the scattering amplitudes $S_{0}$ and $S_{n}$ in Eq. (4) are computed by using the MIEV code ${ }^{13}$ Light extinction spectra for two concentric spheres of size $\alpha$ $=8 \mu \mathrm{m}\left[(\Delta \rho)_{\max }=0.7\right]$, and $\alpha=32 \mu \mathrm{m}\left[(\Delta \rho)_{\max }=2.7\right]$ are shown for the wavelength $500-1000 \mathrm{~nm}$ in vacuum. Even when $(\Delta \rho)_{\max } \sim \pi$, the superposition rule still gives an acceptable approximation to light extinction by a composite scatterer.

Figure 3 displays the intensity of light seattering into different directions (unnormalized phase func-

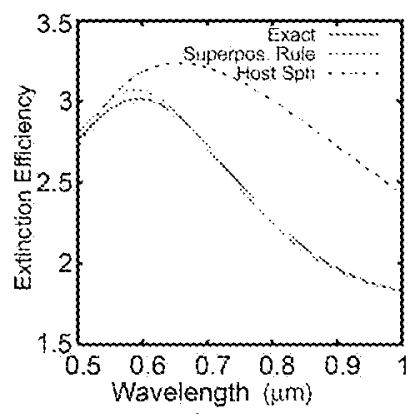

(a)

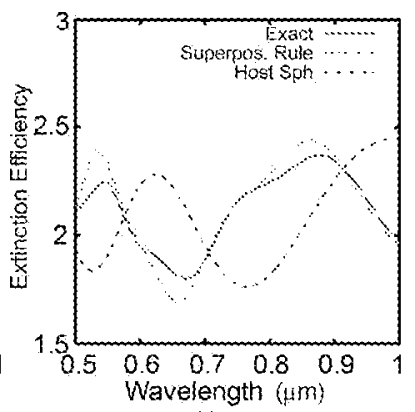

(b)
Fig. 2. (Color online) Extinction efficiencies for various concentric spheres of radius (a) $\alpha=8 \mu \mathrm{m}$ and (b) $\alpha=32 \mu \mathrm{m}$. The ratio of the size of the nucleus over that of the host particle is $\gamma=0.5$. The relative refiactive indices are $m_{0}=1.02$ for the host particle and $m_{1}=1.08$ for the nucleus. The refractive index of the environment is assumed to be 1.33 . The values obtained by using the superposition rule agree well with the exact ones. The extinction efficiencies for the host particle alone are also plotted for comparison.

tion) computed exactly and approximately by using the superposition rule for various concentric spheres. The agreement between the spectra obtained by the superposition rule and the exact ones is excellent.

Light scattering by a composite seatterer with an eccentric inclusion may depend on both the polar and azimuthal angles of scattering. The superposition rule reproduces the phenomenon that a lower refractive index inclusion causes a secondary diffraction ring to appear on its opposite side. The superposition rule can also be used to compute light scattering by a composite particle with a randomly placed inclusion in a straightorward fashion. One example of practical importance is a spherical scatterer with a nucleus randomly placed inside emulating a biological cell. The scattering amplitude function of the composite scatterer can be expressed as

$$
S(\boldsymbol{q})=S_{0}(\boldsymbol{q})+f\left(\boldsymbol{q}, x_{c}\right) S_{n}(\boldsymbol{q})
$$

where $f\left(\boldsymbol{\alpha}, \boldsymbol{r}_{i}\right)=\exp \left[-i \boldsymbol{r}_{i} \cdot \mathbf{q}-i \rho_{\max } \sqrt{1-\left(\xi_{c}^{2}+\eta_{c}^{2}\right) / \alpha^{2}}\right]$ and $\rho_{\max }=2 k\left(m_{0}-1\right) a$ when the nucleus is located at $\mathbf{r}_{c}$. The average phase delay $\bar{\rho}$ has been replaced by the phase delay for the ray passing through the center of the nucleus for simplicity. The scattering amplitude $\bar{S}(w)$ of the composite sphere with a randomly placed inclusion is obtained by performing a configurational average of $f\left(\alpha, r_{c}\right)$ in $\mathrm{Eq},(6)$ over $\mathfrak{w}_{c}$, which yields 

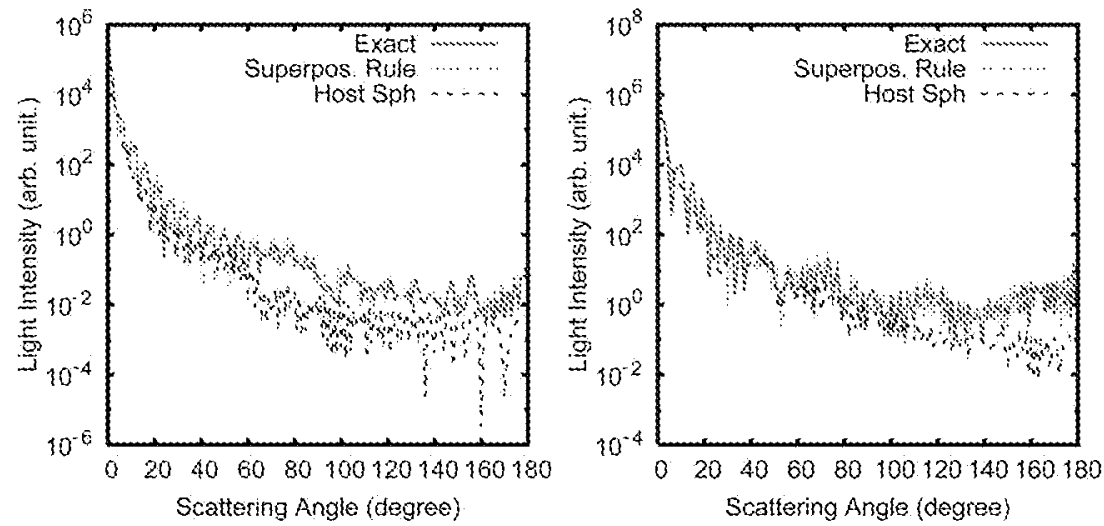

Fig. 3. (Color online) Angular light scattering by various concentric spheres of the same parameters as those in Fig. 2. The wavelength of the incident light is $500 \mathrm{~nm}$. The angular spectra obtained by using the superposition rule agree well with the exact ones. The angular spectra for the bost particle alone are also plotted for comparison.

$$
\begin{aligned}
\overline{f(\boldsymbol{\alpha})}= & 3 \int_{0}^{\pi / 2} \exp \left(-i \rho_{\max } \sqrt{1-\epsilon^{2} \cos ^{2} \tau}\right) \\
& \times J_{0}(q \alpha \epsilon \cos \tau) \cos \tau \sin ^{2} \tau \mathrm{d} \tau,
\end{aligned}
$$

dependent on $\epsilon a$, the maximum displacement of the nucleus from the center of the host. The behavior of $\bar{S}(\boldsymbol{\alpha})$ departs from that of a concentric sphere when $\epsilon>0$. It is noted that the phase function of such a composite particle is determined by $\overline{|S(\boldsymbol{q})|^{2}}=\left|S_{0}(\boldsymbol{\alpha})\right|^{2}$

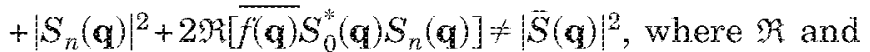
* denote the real part and the complex conjugate, respectively.

The superposition rule provides a simple means to analyze light scattering by a soft complex particle and to quantify the contributions from each individual inclusion. It is, in particular, suited to investigate light scattering by a biological cell and its internal structure (nucleus, mitochondria, and other organelles). Since the scattering amplitude fully determines the (vector) light scattering properties by a scatterer, it is believed the superposition rule about the scattering amplitude will be important to understand light scattering by a composite particle and facilitate noninvasive probing of such particles using light.

The author's e-mail address is mxu@mail.fairfield.edu.

\section{References}

1. H. C. van de Hulst, Light Scattering by Small Particles (Dover, 1981).

2. M. I. Mishchenko, J. W. Hovenier, and L. D. Travis, eds., Light Scattering by Nonspherical Paricles: Theory, Measurements, and Applications (Academic, 1999).

3. M. P. Ioannidou, I. I. Bakatsoula, and D. P. Chrissoulidis, Appl. Opt. 39, 4205 (2000).

4. D. Arifler, M. Guillaud, A. Carraro, A. Malpica, M. Follen, and R. Richards-Kortum, J. Biomed. Opt. 8, 484 (2003).

5. R. Drezek, M. Guillaud, T. Collier, I. Bioko, A. Malpica, C. Macaulay, M. Follen, and R. Richards-Kortum, $J$. Biomed. Opt. 8, 7 (2003).

6. G. Videen, D. Ngo, P. Chylek, and R. G. Pinnick, J. Opt. Soc. Am. A 12, 922 (1995).

7. D. R. Secker, P. H. Kaye, R. S. Greenaway, E. Hirst, D. L. Bartley, and G. Videen, Appl. Opt. 39, 5023 (2000).

8. M. Xu, M. Lax, and R. R. Alfano, Opt. Lett. 28, 179 (2003).

9. M. Xu, Appl. Opt. 42,6710 (2003).

10. Y. Liu, W, P. Arnott, and J. Hallett, Appl. Opt. 37, 5019 (1998).

11. P. Yang, Z. Zhang, B. Baum, H.-L. Huang, and Y. Hu, $J$. Quant, Spectrosc. Radiat. Transf. 89, 421 (2004).

12. The coated sphere Mie code by $\mathrm{C}$. Mätzler, is available at http//www.t-matrix.de/.

13. W. J. Wiscombe, Appl. Opt. 19, 1505 (1980). 